\title{
AVANCES EN LA NOMENCLATURA DE LA FLORA DEL CONO SUR: RANUNCULUS GRISEBACHII (RANUNCULACEAE),UN NOMBRE DE REEMPLAZO PARA RANUNCULUS SARMENTOSUS
}

\author{
Christian A. Zanotti
}

Instituto de Botánica Darwinion, IBODA-CONICET, Labardén 200, Casilla de Correo 22, B1642HYD, San Isidro, Buenos Aires, Argentina; czanotti1979@gmail.com (autor corresponsal).

\begin{abstract}
Zanotti, C. A. 2021. Advances in the nomenclature of the Southern Cone flora: Ranunculus grisebachii (Ranunculaceae), a replacement name for Ranunculus sarmentosus. Darwiniana, nueva serie 9(1): 72-74.

In the framework of the continuous updating of the relational database "Documenta Florae Australis", a new name is proposed here, Ranunculus grisebachii, to replace the illegitimate homonym Ranunculus sarmentosus published by Grisebach in 1879 .
\end{abstract}

Keywords. Homonym; nomenclature; replacement name.

Resumen. Zanotti, C. A. 2021. Avances en la nomenclatura de la flora del Cono Sur: Ranunculus grisebachii (Ranunculaceae), un nombre de reemplazo para Ranunculus sarmentosus. Darwiniana, nueva serie 9(1): 72-74.

En el marco de la actualización permanente de la base de datos relacional "Documenta Florae Australis", se propone aquí un nombre nuevo, Ranunculus grisebachii, para reemplazar al homónimo ilegítimo Ranunculus sarmentosus publicado por Grisebach en 1879.

Palabras claves. Homónimo; nombre de reemplazo; nomenclatura.

Ranunculus L. es el género más numeroso dentro de la familia Ranunculaceae con alrededor de 600 especies de hierbas anuales o perennes, tanto terrestres como acuáticas o semiacuáticas (Tamura, 1993). El género posee una distribución cosmopolita (Lourteig, 1951), aunque la mayor riqueza específica se encuentra en las regiones templadas y frías del hemisferio norte y sur, y en zonas montañosas de los trópicos (Biganzoli \& Zanotti, 2010). En Argentina se registran 26 especies que habitan generalmente en ambientes húmedos, vertientes o pantanos (Lourteig, 1951; Zuloaga et al., 2019).

Las revisiones taxonómicas realizadas por Lourteig (1951, 1956a, b) constituyen la principal contribución al conocimiento sistemático de Ranunculus en América del Sur. En la Argentina, además, el género ha sido tratado en floras regionales (Pontiroli, 1967; Lourteig, 1984; Bacigalupo, 1987; Novara, 1993; Kiesling, 1994) así como en trabajos particulares que citan nuevos registros y ampliaciones en la distribución geográfica (Hernández, 1993; Lehnebach et al., 2009; Biganzoli \& Zanotti, 2010).

En el marco de la actualización permanente de la base de datos relacional "Documenta Florae Australis" (http://www.darwin.edu.ar/Proyectos/ FloraArgentina/fa.htm), el nombre Ranunculus sarmentosus Griseb. publicado por Grisebach (1879) resultó ser un homónimo ilegítimo posterior del nombre publicado previamente por Adams (1834) basándose en una planta proveniente de Rusia (Art. 53.1 del ICN, Turland et al., 2018). 
Desafortunadamente, cabe destacar que la homonimia aquí tratada ha resultado en citaciones erróneas debido a que el nombre Ranunculus sarmentosus Adams fue consignado en el Catálogo de las Plantas Vasculares de Bolivia (Beck \& Shortt, 2014: 1128) y también en el Catálogo de las Plantas Vasculares de las Américas (Ulloa Ulloa et al., 2017, material suplementario: 2176) como el nombre correcto del taxón en cuestión, en lugar de haber utilizado el homónimo posterior correspondiente. En línea con lo expuesto, el objetivo de la presente contribución radica en proponer un nombre de reemplazo (Art. 6.11, Turland, et al. 2018) para Ranunculus sarmentosus.

Ranunculus grisebachii C.A. Zanotti, nom. nov. Nombre reemplazado: Ranunculus sarmentosus Griseb. mut. char. Lourteig, Abh. Königl. Ges. Wiss. Göttingen 24: 13. 1879, nom. illeg. TIPO: Argentina, Tucumán, "prope La Ciénaga", 10-14-I-1874, P.G. Lorentz \& G.H.E.W. Hieronymus 655 [lectótipo GOET 009824!, designado por A. Lourteig, Darwiniana 9(3-4): 477. 1951; probable isolectótipo, CORD 00005794!].

Etimología. El nombre de la especie es dedicado a August H. R. Grisebach (1814-1879), taxónomo alemán que ha contribuido a la descripción de numerosas especies ligadas a la Flora Argentina.

Distribución geográfica. Especie nativa de Argentina y Bolivia. En la Argentina ha sido coleccionada en las Sierras de la Aconquija (Tucumán), las Sierras de Córdoba y en Catamarca, entre los 2000-3100 m s.m. En Bolivia se registra en los Dptos. de Cochabamba y La Paz, en valles secos y en la puna húmeda, entre los 2800-3600 m s.m.

Observaciones. Grisebach (1879) describe que la flor posee cinco pétalos, el gineceo 3-8 carpelos y el receptáculo es glabro. Sin embargo, Lourteig (1951), basado en la observación del tipo y del material adicional examinado, afirma que la flor presenta tres pétalos, el gineceo 10-22 carpelos y el receptáculo es hirsuto-piloso.
Por este motivo, se considera necesario agregar la locución latina "mut. char.", que significa "con los caracteres modificados", ya que en la circunscripción del taxón en cuestión se han introducido cambios en caracteres morfológicos de importancia taxonómica (Rec. 47A1, Turland et al., 2018).

Al momento de publicar la especie, Grisebach (1879) citó un ejemplar coleccionado en Tucumán sin consignar colector ni herbario en donde se encuentra depositado el material original. De acuerdo con Lourteig (1951: 477), el "tipo" de Ranunculus sarmentosus es el ejemplar Lorentz \& Hieronymus 655, actualmente alojado en GOET. Según el Art. 9.10 del ICN (Turland et al., 2018) la indicación de Lourteig (1951) puede considerarse como una designación implícita de tipo (McNeill, 2014; Prado et al., 2015). Respecto al probable isolectótipo depositado en CORD, el ejemplar presenta un intervalo de días en la fecha de colección diferente al del lectótipo seleccionado por Lourteig (“10-17" vs. "10-14” en el lectótipo); por todo lo demás, el material en cuestión coincide con el ejemplar seleccionado por la autora.

\section{AGRADECIMIENTOS}

A Pablo Moroni (IBODA) y al revisor anónimo, quienes mediante sus correcciones y sugerencias contribuyeron significativamente a mejorar el trabajo.

\section{BIBLIOGRAFÍA}

Adams, M. F. 1834. Descriptiones plantarum minus cognitarum sibiriae, praesertim orientalis, quas in itinere Ann. 1805 et 1806 observavit. Mémoires de la Société impériale des naturalistes de Moscou 3: 244-245.

Bacigalupo, N. M. 1987. Ranunculaceae, en N. S. Troncoso \& N. M. Bacigalupo (eds.). Flora Ilustrada de Entre Ríos. Colección Científica del Instituto Nacional de Tecnología Agropecuaria 6(3): 305-321.

Beck, S. J. \& J. Shortt. 2014. Ranunculaceae, en P. M. Jørgensen, M. H. Nee \& S. G. Beck. (eds.). Catálogo de las Plantas Vasculares de Bolivia. Monographs in Systematic Botany from the Missouri Botanical Garden 127: 1128.

Biganzoli, F. \& C. A. Zanotti. 2010. Ranunculus filamentosus (Ranunculaceae), nuevo registro para el noroeste de la Argentina. Darwiniana 48(1): 109-112. 
Grisebach, A. 1879. Symbolae ad Floram Argentinam. Abhandlungen der Königlichen Gesellschaft der Wissenschaften zu Göttingen 24: 1-345.

Hernández, H. M. 1993. Ranunculus parviflorus L. (Ranunculaceae), especie adventicia en la Argentina. Lilloa 38(1):117-119.

Kiesling, R. 1994. Ranunculaceae, en R. Kiesling; M. E. Múlgura \& E. A. Ulibarri (eds.). Flora de San Juan: República Argentina. Buenos Aires. I: 188-197.

Lehnebach, C.; K. Schittek \& L. Iharlegui. 2009. Discovery of the Patagonian species Ranunculus fuegianus Speg. (Ranunculaceae) in the altiplano peatlands of northern Argentina and its lectotypification. Gayana, Botánica 66(2): 286-289.

Lourteig, A. 1951. Ranunculáceas de Sudamérica Templada. Darwiniana 9(3-4): 397-608.

Lourteig, A. 1956a. Ranunculáceas de Sudamérica Tropical. Memoria de la Sociedad de Ciencias Naturales La Salle 16(43): 19-88.

Lourteig, A. 1956b. Ranunculáceas de Sudamérica Tropical. Memoria de la Sociedad de Ciencias Naturales La Salle 16(44): 125-228.

Lourteig, A. 1984. Ranunculaceae, en M. N. Correa (ed.). Flora Patagónica, Colección Cientifica del Instituto Nacional de Tecnología Agropecuaria 8(4a): 284-322.

McNeill, J. 2014. Holotype specimens and type citations: General issues. Taxon 63: 1112-1113. DOI: https://doi. org/10.12705/635.7

Novara, L. J. 1993. Ranunculaceae, en L. J. Novara (dir.). Flora del Valle de Lerma. Aportes Botánicos de Salta, Ser. Flora 2(6): 1-17.
Pontiroli, A. 1967. Ranunculaceae, en A. L. Cabrera (ed.). Flora de la Provincia de Buenos Aires, Colección Cientifica del Instituto Nacional de Tecnología Agropecuaria 4(3): 243-257.

Prado, J.; R. J. Hirai \& R. C. Moran. 2015. (046-048) Proposals concerning inadvertent lectotypifications (and neotypifications). Taxon 64: 651. DOI: http://dx.doi. org/10.12705/643.29

Tamura, M. 1993. Ranunculaceae, en K. Kubitzki; J. G Rowher \& V. Bittrich (eds.). The Families and genera of vascular plants. Berlín: Springer-Verlag. II: 563-583. DOI: https:// doi.org/10.1007/978-3-662-02899-5 67

Turland, N. J.; J. H. Wiersema, F. R. Barrie, W. Greuter, D. L. Hawksworth, P. S. Herendeen, S. Knapp, W. H. Kusber, D. Z. Li, K. Marhold, T. W. May, J. Mcneill, A. M. Monro, J. Prado, M. J. Price \& G. F. Smith (eds.). 2018. International Code of Nomenclature for algae, fungi, and plants (Shenzhen Code). Regnum Veg. 159. Koeltz Botanical Books, Glashütten. DOI: https://doi.org/10.12705/Code.2018

Ulloa Ulloa, C.; P. Acevedo-Rodríguez, S. G. Beck, M. J. Belgrano, R. Bernal González, P. E. Berry, L. Brako, M. Celis, G. Davidse, S. R. Gradstein, O. Hokche, B. León, S. León-Yánez, R. E. Magill, D. A. Neill, M. H. Nee, P. H. Raven, H. Stimmel, M. T. Strong, J. L. Villaseñor Ríos, J. L. Zarucchi, F. O. Zuloaga \& P. M. Jørgensen. 2017. An integrated assessment of vascular plants species of the Americas. Science 358: 1614-1617. DOI: https://doi. org/10.1126/science.aao0398

Zuloaga, F. O.; M. J. Belgrano \& C. A. Zanotti. 2019. Actualización del Catálogo de las Plantas Vasculares del Cono Sur. Darwiniana, nueva serie 7(2): 208-278. DOI: https://doi.org/10.14522/darwiniana.2019.72.861 